\title{
Tailoring Dispersion and Eigenfield Profiles of Plasmonic Surface Lattice Resonances
}

\author{
Aimi Abass, ${ }^{*}{ }^{\dagger}, \#$ Said Rahimzadeh-Kalaleh Rodriguez, ${ }^{*}, \#$ Jaime Gómez Rivas, ${ }^{\ddagger}$, and Bjorn Maes ${ }^{\perp, \|}$ \\ ${ }^{\dagger}$ Solar Cells Group, Department of Electronic and Information Systems (ELIS), Ghent University, Sint-Pietersnieuwstraat 41, B-9000 \\ Ghent, Belgium \\ ${ }^{\ddagger}$ Center for Nanophotonics, FOM Institute AMOLF, c/o Philips Research Laboratories, High Tech Campus 4, 5656 AE Eindhoven, \\ The Netherlands \\ ${ }^{\S}$ COBRA Research Institute, Eindhoven University of Technology, P.O. Box 513, 5600 MB Eindhoven, The Netherlands \\ ${ }^{\perp}$ Micro- and Nanophotonic Materials Group, Faculty of Science, University of Mons, Place du Parc 20, B-7000 Mons, Belgium \\ "Photonics Research Group, Department of Information Technology (INTEC), Ghent University-imec, Sint-Pietersnieuwstraat 41, \\ B-9000 Ghent, Belgium
}

\begin{abstract}
We investigate the radiative coupling between localized surface plasmon resonances (LSPRs) and Rayleigh anomalies (RAs) in periodic arrays of metallic nanorods with varying dimensions but equal lattice constants. The dimensions of the nanorods determine the energy and line width of the LSPR and, thus, enable tailoring of the mixed LSPR-RA states: surface lattice resonances (SLRs). We present variable angle light extinction experimental spectra for five arrays with different nanorod width and explain our results with numerical simulations. The numerical simulations are done for driven and undriven systems, with the latter revealing the SLR eigenmode properties for the first time. We provide a plane wave model that interprets the near- and far-fields of these eigenmodes, describing the intricate behavior of confinement and radiative loss versus in-plane momentum. The SLR line width, band gap associated with the coupled modes, and field extension into the surrounding dielectric are tunable via the dimensions of the nanorods.
\end{abstract}

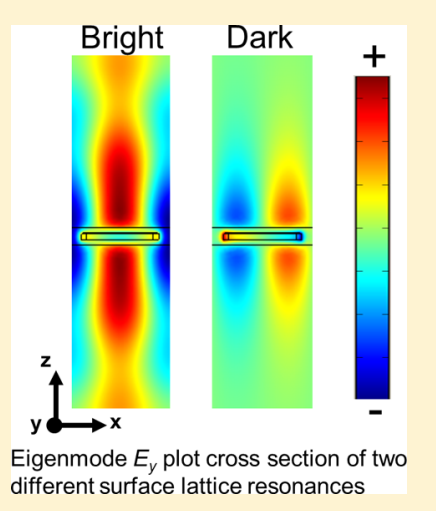

KEYWORDS: localized surface plasmon resonance, Rayleigh anomaly, Fano resonance, surface lattice resonance, eigenmode profiles, plane wave model

S urface electromagnetic modes in metallic nanostructures have attracted a broad scientific interest, as they lead to a rich optical response that is tunable by geometrical design. ${ }^{1}$ When the electromagnetic fields associated with two distinct surface modes overlap spatially, the modes can couple and their properties (e.g., their energy dispersion and line width) are altered according to the coupling strength. Coupled surface modes have been observed in metallic gratings, ${ }^{2-5}$ subwavelength hole arrays, ${ }^{6-9}$ nanoslit arrays, ${ }^{10,11}$ stacked plasmonic nanowire arrays, ${ }^{12}$ and particle arrays coupled to waveguide modes. ${ }^{13,14}$ In recent years periodic arrays of metallic nanoparticles have attracted much attention for their ability to support collective resonances arising from the radiative coupling between localized surface plasmon resonances (LSPRs) and waves diffracted in the plane of the array [Rayleigh anomalies (RAs)]. ${ }^{15-25}$ These collective resonances, also known as surface lattice resonances (SLRs), have quality factors $Q$ depending on the number of particles in the array, ${ }^{26}$ and their dispersion and line width are tunable via the particle geometry and lattice constant. ${ }^{23,25}$ Recent work has shown the relevance of SLRs for enhancing the spontaneous emission of luminescent molecules or quantum dots within the vicinity of the array, ${ }^{27-29}$ for lasing, ${ }^{30}$ and for refractive index sensing. ${ }^{31}$
Despite the numerous fundamental and applied studies concerning SLRs, their general properties in terms of the coupling conditions between the participating plasmonic and photonic modes are limitedly explored. In this sense, remarkable insight was obtained by Barnes and co-workers regarding the coupling between distinct surface plasmon polaritons in metallic sinusoidal gratings. ${ }^{3}$ However, the analysis contained therein is not easily extended to more complex plasmonic structures, which renders difficult the emergence of a simple, intuitive explanation on the characteristics of the coupled surface modes. Another important study was presented recently by Teperik and Degiron, ${ }^{25}$ who explained how the SLR energy and line width (at normal incidence only) can be tuned by tailoring the polarizability tensor describing the nanoparticles. While such an approach is appealing from a theoretical standpoint, experimental implementation is difficult, as it requires a priori knowledge of how the particle dimensions (the experimentalist's turning knob) relate to the polarizability tensor.

It is the aim of this work to elucidate how the SLR characteristics depend on the spectral properties of the 

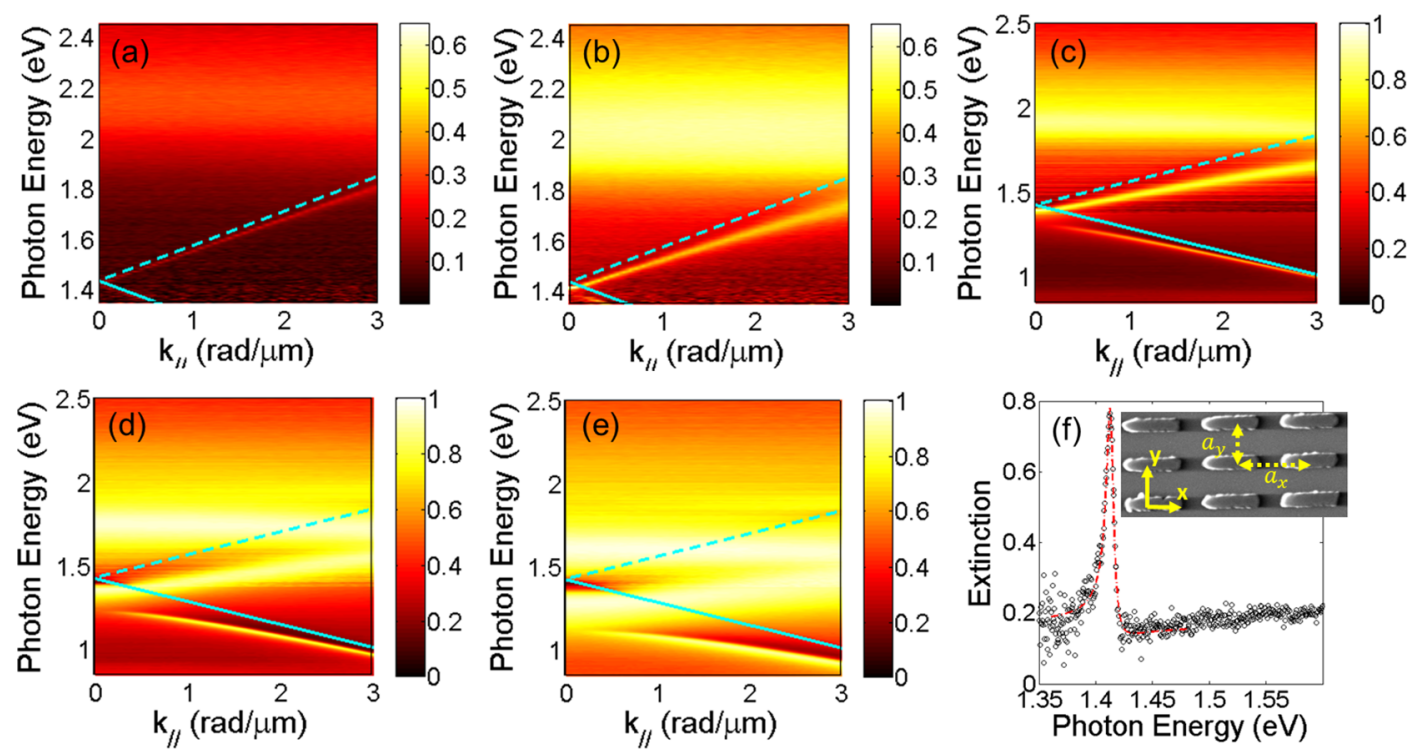

Figure 1. Experimentally measured extinction for (a) $w=70 \mathrm{~nm},(\mathrm{~b}) w=110 \mathrm{~nm},(\mathrm{c}) w=160 \mathrm{~nm},(\mathrm{~d}) w=200 \mathrm{~nm}$, and (e) $w=230 \mathrm{~nm}$. (f) Experimental extinction at $k_{/ /}=0$ for $w=110 \mathrm{~nm}$ array, fitted with a Fano resonance (red dashed lines) for the upper SLR. The inset in (f) shows the SEM picture of the nanorod array with $w=110 \mathrm{~nm}$.

interacting RAs and LSPRs, which are in turn determined by simple structural design. We focus on nanorod arrays where RAs associated with two diffraction orders $[(+1,0)$ and $(-1,0)]$ interact with a single first-order LSPR. Experimental extinction spectra of arrays with nanorods of different dimensions are investigated. The nanorod dimensions determine the energy and line width of the LSPR and, thus, the detuning with respect to the RAs in arrays with fixed periodicity. We focus on the case of light polarized along the width of the nanorod where the LSPR red-shifts and broadens for increasing nanorod width due to the depolarization field along this dimension. ${ }^{32}$ Our work analyzes how this changes the coupling of the LSPR to the RAs. We shed light on the physics through transmission and eigenmode simulations using finite element based methods. The eigenmode numerical simulations illustrate how the SLR field profile varies for different LSPR-RA energy detunings, including changes in in-plane momentum. Finally these eigenmode profiles are described by a straightforward plane wave model, elucidating the subtle interferences at play.

\section{EXPERIMENTAL RESULTS}

We fabricated five gold nanorod arrays with varying nanorod widths $(w)$ of $70,110,160,200$, and $230 \mathrm{~nm}$ (in the $y$ direction) but an equal nanorod length of $450 \mathrm{~nm}$ (in the $x$ direction) on a silica substrate using electron beam lithography. The arrays have dimensions of $1.5 \times 1.5 \mathrm{~mm}^{2}$ and lattice constants $a_{x}=600 \mathrm{~nm}$ and $a_{y}=300 \mathrm{~nm}$. The nanorods have an approximately rectangular shape in the plane of the array and a height of $40 \pm 2 \mathrm{~nm}$. The rod width was tuned by varying the exposure dose of the electron beam. The tolerances of the inplane dimensions are on the order of nanometers. The arrays were embedded in a uniform surrounding medium by placing a silica superstrate preceded by $n=1.45$ index matching fluid to ensure good optical contact.

Figure $1 \mathrm{a}-\mathrm{e}$ shows the extinction of $y$-polarized light, defined as $1-T_{0}$, with $T_{0}$ the zeroth-order transmittance, for the five arrays described above. The extinction is displayed in color as a function of the incident photon energy and component of the wave vector parallel to the surface in the $x$ direction, which is given by $k_{/ /}=(E / \hbar c) \sin (\theta)$, with $\theta$ the angle of incidence from the normal. The samples were rotated around the $y$-axis, while the $y$-polarized collimated beam from a halogen lamp impinged onto the sample, probing the short axis of the nanorods.

The dispersionless and broad extinction peak seen on the high-energy side of the extinction spectra for all five arrays corresponds to the excitation of LSPRs in the individual nanorods. The cyan solid and dashed lines indicate the $(-1,0)$ and $(+1,0)$ RAs, respectively. The RAs are solutions to the equation $E_{ \pm}=(\hbar c / n)\left|k_{/ /}+m G_{x}\right|$, where $m= \pm 1$ is the order of diffraction, and $G_{x}=2 \pi / a_{x}$ is the $x$-component of the reciprocal lattice vector. The coupling of LSPR to the RAs yields the upper and lower SLRs. The SLRs are dispersive bands in extinction with variable line width, following the dispersion of the RAs on the low-energy side. Notice that, at $k_{/ /}=0$, the upper SLR is bright (i.e., it is excited strongly by the incident plane wave), while the lower SLR is dark (i.e., it is not excited at all). The bright/dark SLR mutual coupling leads to an energy stop-gap near the intersection of the RAs. ${ }^{23}$ We note that this is not a complete band gap, since it exists only for light polarized parallel to the short axis of the nanorods ( $y$ direction) and with an in-plane wave vector component parallel to the long axis of the nanorods. For light polarized parallel to the long axis of the nanorods, the dipolar LSPR lies at lower energies than the $( \pm 1,0)$ diffraction orders at normal incidence, which results in a weak diffractive coupling. ${ }^{20}$ On the other hand, for an in-plane wave vector component parallel to the short axis of the nanorods, the $( \pm 1,0)$ Rayleigh anomalies are degenerate, leading to degenerate $( \pm 1,0)$ SLRs and therefore to the absence of a gap. ${ }^{33}$

The measurements in Figure 1a-e show a clear correlation between the SLR characteristics and the spectral properties of the LSPR. As the LSPR broadens and approaches the RAs in energy upon increase of $w$, the SLRs shift toward lower energies, their line widths broaden, and the gap between them widens. Additionally, the SLR dispersion deviates more strongly from the associated RA for increasing $w$. This indicates that the coupling strength between LSPRs and RAs increases. The broadening of the SLR line width, which implies increased 

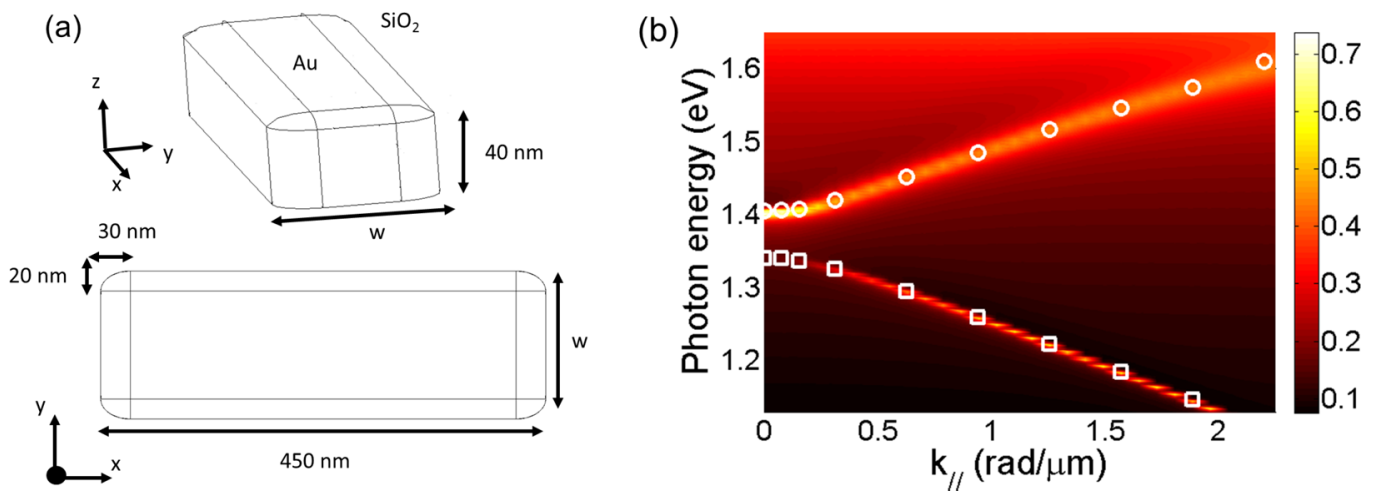

Figure 2. (a) Diagram of the simulated nanorods. (b) Simulated extinction (color plot) dispersion for $w=110 \mathrm{~nm}$. White markers indicate the eigenfrequencies of the SLR modes obtained through eigenfrequency calculations. Circle and square markers indicate data points for upper and lower SLR, respectively.

losses, can be understood on the basis of the increased influence from the lossy LSPR. The effect of LSPR-RA energy detuning can also be observed in the SLR dispersion of each array. As $k_{/ /}$increases, the detuning between the upper SLR and LSPR diminishes. Consequently, the SLR line width broadens and its peak energy deviates more pronouncedly from the $(+1,0)$ RA. In contrast, the lower SLR becomes narrower and approaches the $(-1,0) \mathrm{RA}$ as it becomes more detuned from the LSPR.

The SLRs can exhibit relatively narrow line widths for plasmonic-type resonances. To obtain the line widths of these SLRs, we fit the extinction measurements with a Fano model of the form $F=A_{0}+A\left[\left(q \alpha / 2+E-E_{\mathrm{o}}\right)^{2} /\left[\left(E-E_{0}\right)^{2}+(\alpha / 2)^{2}\right]\right.$. $A_{0}, A, q, \alpha$, and $E_{0}$ are the fitting parameters, which we determine by a least-squares method. Figure if shows the Fano fit of the upper SLR at $k_{/ /}=0$ for $w=110 \mathrm{~nm}$, from which we obtain the line width $(\alpha)$ to be $8.29 \pm 0.56 \mathrm{meV}$ and resonant frequency $\left(E_{0}\right)$ to be $1.413 \mathrm{eV} \pm 0.269 \mathrm{meV}$. The Fano shape of SLRs can be expected as they arise from the coupling between a broad LSPR and narrow RA resonances. ${ }^{23,34}$ These resonances, commonly arising from a broad resonance interacting with narrow resonances, have been found in various other plasmonic systems. ${ }^{34-36}$

\section{NUMERICAL RESULTS}

Eigenmode and Transmission Calculations. By finite element method simulations (COMSOL), we now examine the SLR characteristics in the presence and absence of a driving optical field. In the transmission simulations, we use plane wave illumination coming in at various angles as the driving field to obtain the extinction spectra. The extinction was calculated as 1 - $T_{0}$, with $T_{0}$ the zeroth-order transmission obtained by Fourier decomposition. Figure $2 \mathrm{a}$ shows a sketch of the nanorods in the simulated arrays. Figure $2 b$ shows the simulated extinction of the $w=110 \mathrm{~nm}$ array. Our simulations capture well the SLR characteristics observed in the measurements, and similar agreement was obtained for other arrays. The SLR peak energies obtained from the transmission simulations serve as an initial guess and check for the eigenenergies in the undriven case obtained from eigenmode calculations. With the eigenmode simulations, we focused on finding the SLRs that are Bloch modes that propagate in the plane of the array along the $x$ direction. The SLR eigenenergies calculated at a few values of $k_{/ /}$are plotted as white circles in Figure $2 \mathrm{~b}$. The good agreement of the SLR dispersion obtained with eigenmode and transmission simulations confirms that the obtained Bloch modes are indeed SLRs.

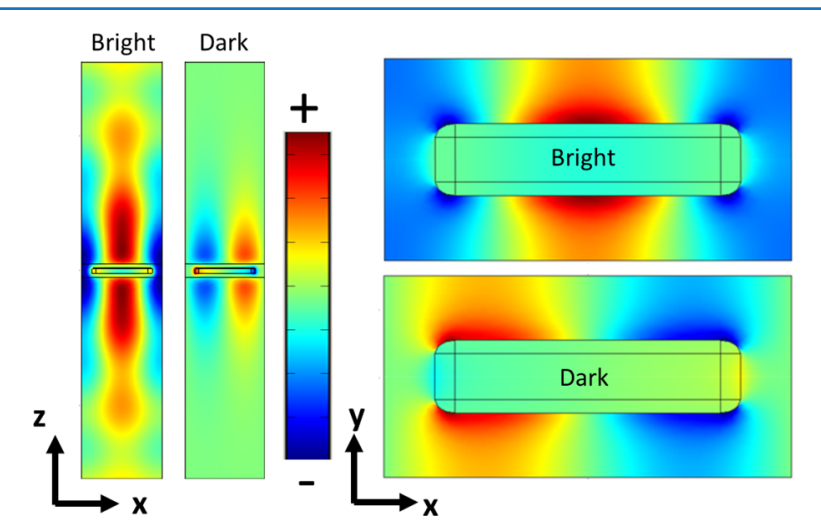

Figure 3. Eigenmode $E_{y}$ plot cross sections of bright and dark modes at the $x z$ - and $x y$-plane through the center of the nanorods for $w=110$ $\mathrm{nm}$ at $k_{/ /}=0$.

Having validated our simulations, we now examine the SLR eigenmode field profiles. The $y$-component of the eigenfield, $E_{y}$ is shown in Figure 3 for the upper and lower SLRs at $k_{/ /}=0$ in the $w=110 \mathrm{~nm}$ array. In both planes $(x z$ and $x y$ ) intersecting the unit cell at its center, it is shown that the eigenfield $E_{y}$ has even parity for the upper SLR and odd parity for the lower SLR (with respect to the $y z$-plane through the particle center). The odd parity of the lower SLR renders the mode dark at normal incidence; that is, it cannot be excited by an incoming plane wave. On the other hand, the upper SLR clearly has a field profile that is not strictly confined to the nanorod, as it has a radiative component unlike the lower SLR. The symmetries of the upper and lower SLR eigenfields are the same for arrays with different $w$ (other dimensions fixed), but the SLR eigenenergies change. In Figure 4 we show how, as $w$ increases and the LSPR-RA energy detuning decreases, the SLRs are shifted to lower energies and the gap widens, in agreement with our measurements $\left(k_{/ /}=0\right.$ in Figure $\left.1 \mathrm{a}-\mathrm{e}\right)$.

In Figure 5 we present the magnitude of the total SLR eigenfields for arrays with different $w$. The upper and lower SLR are shown in Figure 5a and b, respectively. The near-field confinement of both SLRs is enhanced as $w$ increases. This effect is more clearly visible for the lower SLR, as this mode lacks a radiative component. In contrast, the upper SLR retains a radiative component for all $w$. Concentrating on the spatial 


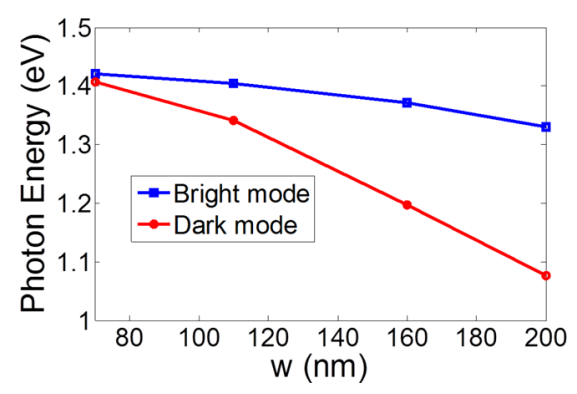

Figure 4. SLR energy dependence on nanorod width at $k_{/ /}=0$ obtained from eigenmode calculations.

region away from the nanorods in Figure 5a, we observe that the magnitude of the eigenfield increases as $w$ increases. Since the fields in this region are mostly radiative in character, it follows that the radiative portion of the total loss also increases with increasing $w$ for the upper SLR. This effect, in combination with the increased near-field confinement, which naturally leads to larger ohmic losses, can thus be expected to increase the line width of the upper SLR at $k_{/ /}=0$ in the driven system. The latter observation is very clear from the experiments (Figure 1a-e).

To further elucidate the trends in Figure 5, we show cross sections of the normalized eigenfield magnitude in Figure 6. The cross sections are taken along the dashed line in Figure 5 and normalized to the first maximum encountered in the dielectric medium. The upper SLR eigenfields in Figure 6a display oscillations due to interference between the near-field and far-field (radiative) components of the eigenmode. In the far-field regime away from the nanorods, the magnitude reaches a "plateau". This asymptotic behavior can be seen in Figure 6a for the nanorod array of $w=160 \mathrm{~nm}$ starting at a distance of 1.5 $\mu \mathrm{m}$ and is more apparent for arrays of wider nanorods. The radiative component of the upper SLR dominates in the region where this plateau sets in. The plateau indicates coupling of the upper SLR to a single radiative plane wave channel, as will be demonstrated more clearly later in the discussion of radiative losses and in the Plane Wave Model section. The onset of the plateau is more clearly visible for wider nanorods, as it occurs at shorter distances away from the nanorods due to the near-field portion of the mode being more tightly confined. The physics are simpler for the lower SLR eigenfield, which decreases monotonously in the dielectric medium as shown in Figure $6 \mathrm{~b}$. The inset in Figure $6 \mathrm{~b}$ shows a plot of the spatial extent of the upper and lower SLR eigenmodes as a function of $w$. The mode extension is taken to be the distance where the eigenfield magnitude drops to $1 / e$ for the first time after the normalization point. There, it can be observed that both SLR eigenmodes become increasingly confined for wider nanorods, again consistent with increasing losses and experimentally observed increasing line widths for the bright mode at $k_{/ /}=0$ in Figure 1.

Having established how the nanorod width influences the SLR eigenmode characteristics at $k_{/ /}=0$, we now examine the dependence on $k_{/ /}$. Naturally, this dependence is different for the upper and lower SLR eigenmodes. Figure 7a shows a plot of the total electric eigenfield magnitude (color plot) and Poynting vector (arrows) for $w=110 \mathrm{~nm}$ at different $k_{/ /}$for the upper SLR. Therein, we identify different regimes depending on the value of $k_{/ /}$. In the low-momentum regime $\left(k_{/ /} \leq 0.16 \mathrm{rad} / \mu \mathrm{m}\right)$, the eigenfield magnitude far away from the nanorods $(>1 \mu \mathrm{m})$ increases relative to the magnitude in the near-field as $k_{/ /}$increases. As in the case of the nanorod widening, this apparent increase of far-field magnitude suggests an increase in radiative losses at intermediate $k_{/ /}$, which will be discussed later. For higher momenta $\left(k_{/ /} \geq 0.16 \mathrm{rad} / \mu \mathrm{m}\right)$, the eigenfield magnitude away from the nanorods $(>1 \mu \mathrm{m})$ decreases relative to the magnitude in the near-field. This trend at high $k_{/ /}$is due to the increase of near-field confinement as the SLR approaches the LSPR and obtains a more localized nature.

As before, the eigenfield profile evolution of the lower SLR is more monotonic (Figure $7 \mathrm{~b}$ ). As $k_{/ /}$increases, the odd symmetry of the lower SLR is broken. This leads the mode out of darkness, as the radiative component increases and the mode becomes less confined. The latter effect is also connected with the fact that the lower SLR detunes from the LSPR and approaches the $(-1,0) \mathrm{RA}$ as $k_{/ /}$increases. The near- and far-
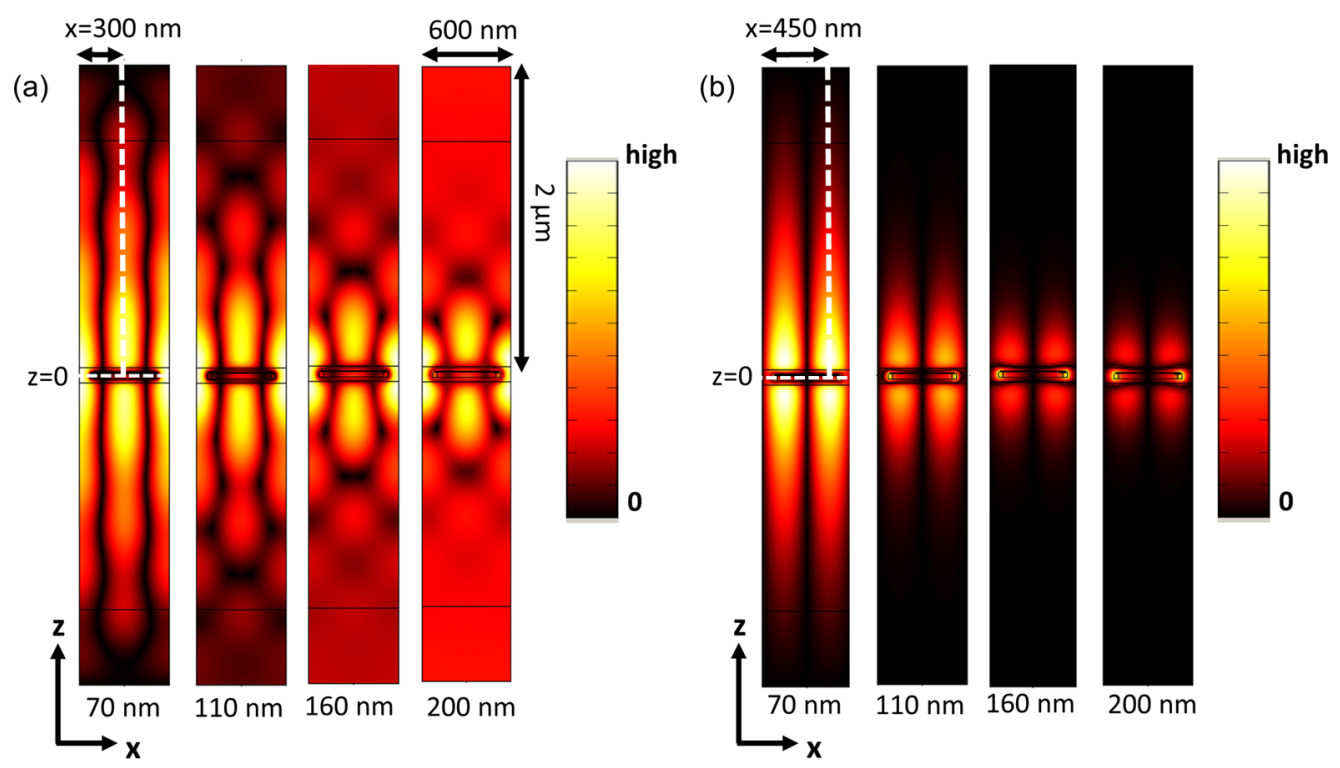

Figure 5. $x z$ cross section plot through the center of the nanorod of the eigenmode $|E|$ profile of (a) bright and (b) dark modes for different $w$ at $k_{/ /}$ $=0$. Each $|E|$ plot has a different normalization individually. The dimension listed is the nanorod width $(w)$. 

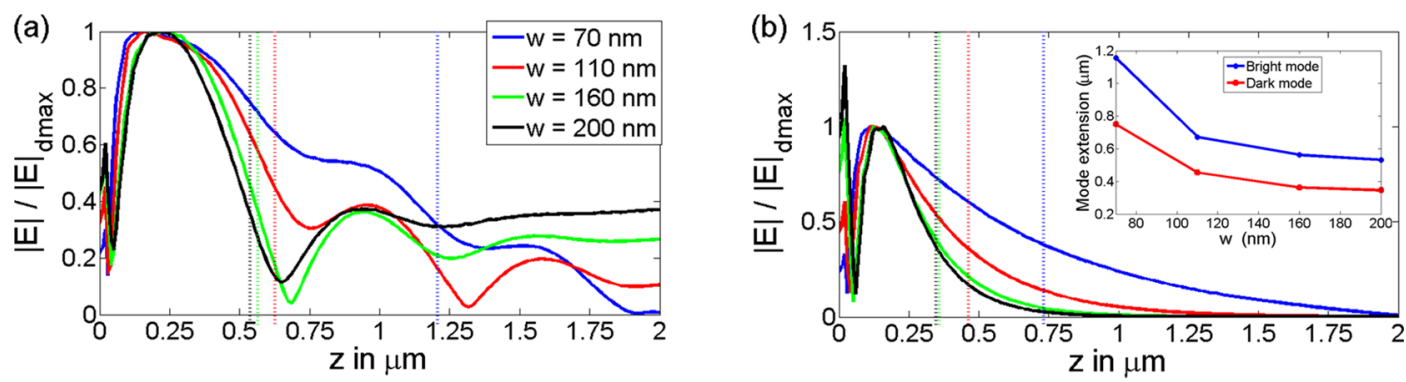

Figure 6. Normalized cross-section of (a) bright mode and (b) dark mode field profile at $k_{/ /}=0$. The vertical dotted lines indicate the field extension for each array according to the definition described in the text. The inset of (b) shows the plot of the dark and bright mode field extension.
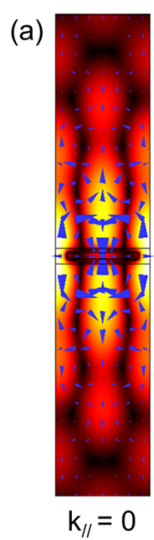

(b)
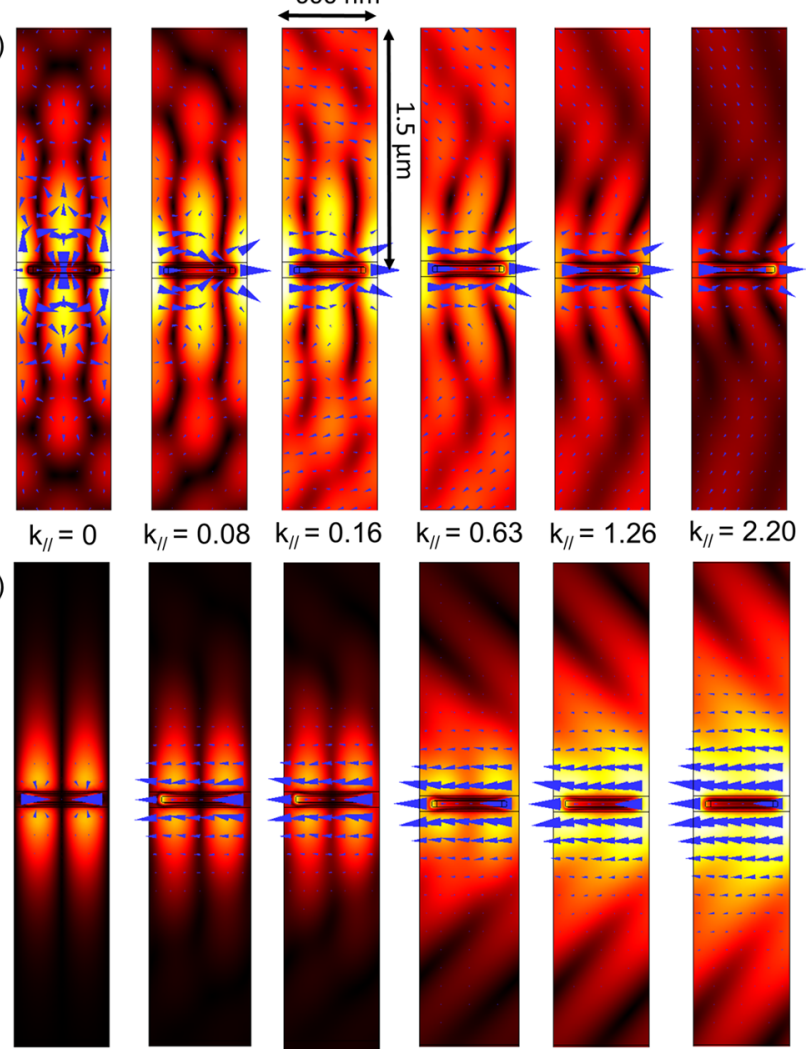

Figure 7. (a) Upper SLR and (b) lower SLR modal field profile evolution as $k_{/ /}$is increased for $w=110 \mathrm{~nm}$ ( $x z$ cross section through the center of the nanorod). The shown $k_{/ /}$values are in units of rad/ $\mu \mathrm{m}$ and directed to the right. The blue arrows indicate the Poynting vector.

field properties of the SLR modes are discussed in detail in the next section using a simple plane wave expansion model.
Regarding the Poynting vector, we observe that in the nearfield region it becomes increasingly parallel to the plane of the array as $k_{/ /}$increases for both upper and lower SLRs (Figure 7). However, the Poynting vector of the lower SLR is antiparallel to the direction of $k_{/ /}$. Therefore, it can be recognized that the upper and lower SLRs correspond to surface waves with counter-propagating group velocities (Poynting vectors have opposite directions) but parallel phase velocities (the wave vectors $k_{/ /}$are parallel in Figure 7).

In order to investigate the different regimes observed in the eigenfield profile of Figure 7 in more detail, the relative radiative portion of the loss is numerically calculated as

$$
\gamma_{\text {relative }}^{\text {rad }}=\frac{\gamma_{\text {rad }}}{\gamma_{\text {rad }}+\gamma_{\text {ohmic }}}
$$

where $\gamma_{\text {relative }}^{\text {rad }}$ is the relative radiative loss portion, $\gamma_{\text {rad }}$ is the radiative loss that is calculated by integrating the power leaving the nanorod arrays, and $\gamma_{\text {ohmic }}$ is the ohmic loss in the metal nanorods. In Figure 8a, we plot $\gamma_{\text {relative }}^{\text {rad }}$ of the upper SLR as a function of $k_{/ /}$for three arrays with different nanorod widths. For every array $\gamma_{\text {relative }}^{\text {rad }}$ first decreases until a certain $k_{/ /}$before suddenly increasing again to reach a maximum. The $k_{/ /}$point where the sudden transition occurs is the point where an additional radiative loss channel starts to become available. This additional radiative loss channel is the outcoupling to the $(-1,0)$ diffraction order plane waves that only becomes nonevanescent when the condition $\left|k_{/ /}-G_{x}\right| \leq n k_{0}$ is satisfied. This condition is satisfied at higher $k_{/ /}$starting at the crossing point between the upper SLR with the RA $(-1,0)$. The cusp $k_{/ /}$ point depends on the nanorod width, as changing the width shifts the upper SLR dispersion. After the minimum $\gamma_{\text {relative }}^{\text {rad }}$ increases rapidly with $k_{/ /}$and reaches a maximum before slowly decreasing again. The radiative loss portion decreases again at high $k_{/ /}$due to ohmic loss becoming dominant as the upper SLR approaches the LSPR. The radiative loss portion of the lower SLR is plotted as a function of $k_{/ /}$in Figure $8 \mathrm{~b} . \gamma_{\text {relative }}^{\mathrm{rad}}$ for
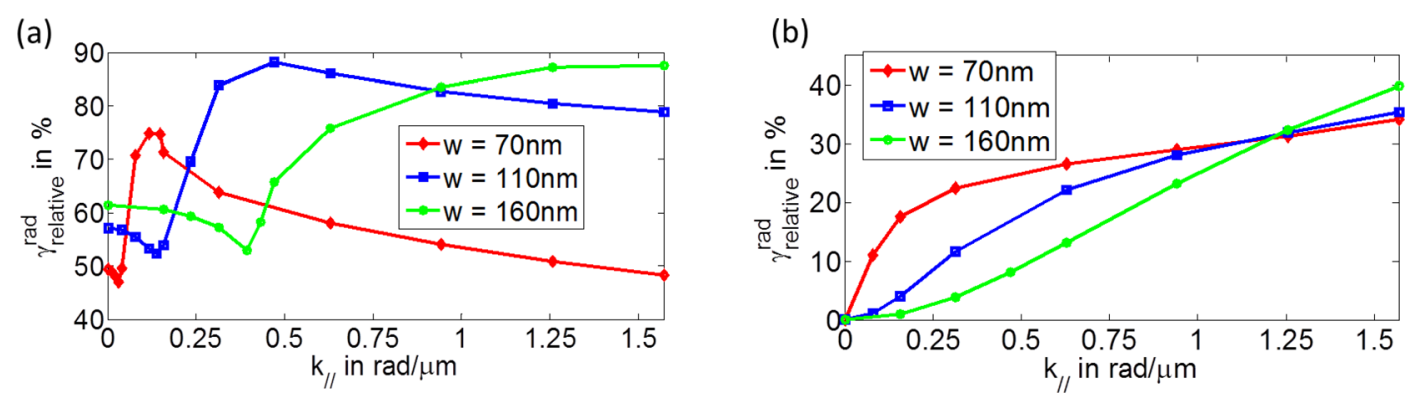

Figure 8. Relative radiative loss portion of the (a) upper SLR and (b) lower SLR for different nanorod widths. 
the lower SLR is zero at $k_{/ /}=0$, and it increases as $k_{/ /}$increases. The slower increase of $\gamma_{\text {relative }}^{\text {rad }}$ at low $k_{/ /}$for arrays with wider nanorods is due to the fact that there is a smaller detuning between the lower SLR and the LSPR, which makes the SLR dispersion in the low $k_{/ /}$regime flatter and its ohmic loss more dominant.

Plane Wave Model. We propose a simple plane wave model, which provides an intuitive basis for the near- and farfield properties of the calculated eigenmodes. Combined with the simulations one obtains a global picture of the engineering possibilities, e.g., for judicious shaping of the near-fields.

The model gives a simple expression for the field in the uniform space above (or below) the nanorods. By exploiting the Bloch character of the eigenmode, we obtain

$$
\psi(x, z)=\sum_{m} a_{m} \mathrm{e}^{i z k_{z}} \mathrm{e}^{i x\left(k_{/ /}+m G_{x}\right)}
$$

where the $z$ dependence stems from the uniform space in the $z$ direction. ${ }^{37}$ Each integer order $m$ corresponds to a particular propagation constant component along $x\left(k_{x}=k_{/ /}+m G_{x}\right)$ and along $z\left(k_{z}=\left(n^{2} k_{0}^{2}-k_{x}^{2}\right)^{1 / 2}\right)$. We neglect the vectorial character, the material loss component, and the $y$ dependence. In addition, we already obtain a characteristic picture of the modes by considering only three orders: $m=0,+1,-1$. The eigenmode shapes are mainly determined by the particular orders that are present, by their symmetry, and by their radiative $\left(k_{z}\right.$ real, "above the light line", $\left.\left|k_{x}\right|<n k_{0}\right)$ or evanescent character ( $k_{z}$ imaginary, "below the light line", $\left.\left|k_{x}\right|>n k_{0}\right)$.

We now propose values for the Fourier amplitudes $a_{0}, a_{+1}$, and $a_{-1}$, based on basic characteristics of the modes. This is not an exact determination by calculation, as this would entail another simulation, as done in the previous section. However, it provides for insight into the underlying Bloch structure of what was rigorously calculated. Note that the dispersion (the values of $k_{0}$ and $\left.k_{/ /}\right)$is considered as a given, determined by the eigenmode simulations (Figure $2 b$ ).

The simplest case is for the lower SLR, when $k_{/ /}=0$. The antisymmetry requires that $a_{0}=0$ and $a_{+1}=-a_{-1}$, so we are limited to only one option $\left(a_{0}, a_{+1}, a_{-1}\right)=(0,1,-1)$. The resulting mode shape $(|\psi|$ in Figure 9a) is similar to the simulated one (Figure $5 \mathrm{~b}$, for $w=110 \mathrm{~nm}$ ). In this case the orders +1 and -1 are both evanescent along $z$. The absence of order 0 , dictated by symmetry, implies that there is no coupling to radiation. Thus, there is no component above the light line, which indeed renders the mode "dark", and the far-field becomes zero for large $z$.

Next we consider the upper SLR, at $k_{/ /}=0$. The even symmetry requires $a_{+1}=a_{-1}$. The value of $a_{0}$ quantifies the radiative component, as again it is the only plane wave above the light line, but it also determines the strength of the central lobe compared to the side-lobes. We plot $|\psi|$ for a choice $\left(a_{0}, a_{+1}, a_{-1}\right)=(0.2,1,1)$ in Figure 9b, which we compare to the simulated profile (Figure 5a, for $w=110 \mathrm{~nm}$ ). The near-field shape with the larger central lobe is created by the nonzero $a_{0}$. In addition, this radiative order 0 provides for the "bright" character of the mode, with nonzero magnitude in the far-field.

The situation becomes more complex for $k_{/ /} \neq 0$. For the lower SLR, for moderate $k_{/ /}>0$, the modes +1 and -1 remain evanescent, as the dispersion curves downward. Therefore, as the mode becomes bright, a nonzero $a_{0}$ is required. We choose $\left(a_{0}, a_{+1}, a_{-1}\right)=(0.5,1,-1)$ in Figure 9c, to compare with Figure $7 \mathrm{~b}$, for $k_{/ /}=1.26 \mathrm{rad} / \mu \mathrm{m}$ with $k_{0}=6.22 \mathrm{rad} / \mu \mathrm{m}$. The interference between the three orders clearly recreates the (a)

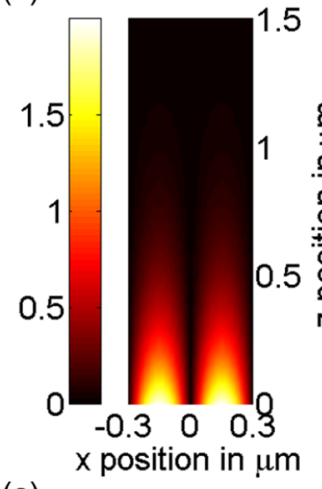

(c)

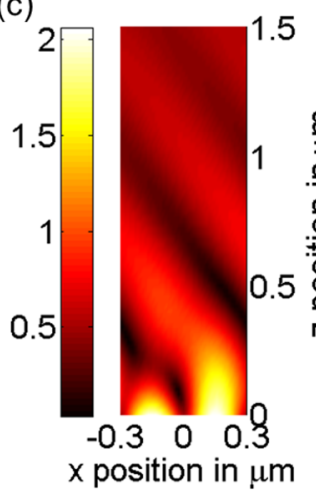

(b)

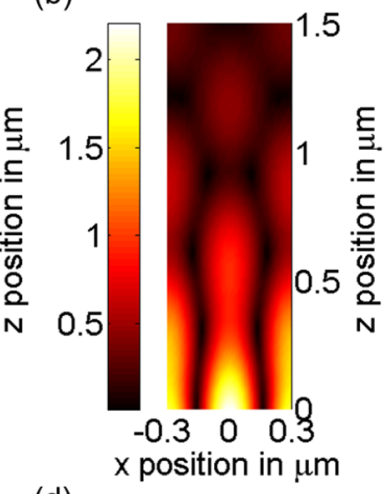

(d)

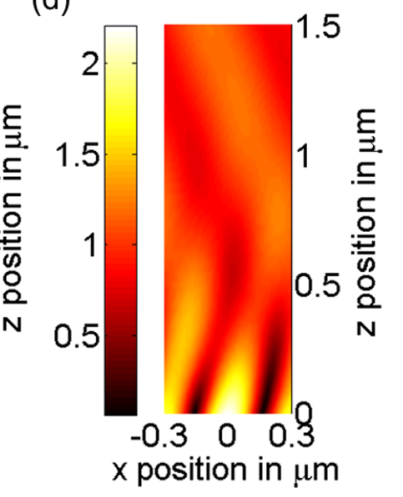

Figure 9. Plane wave model amplitude ly/ for (a) lower SLR $\left(k_{/ /}=0\right.$, $\left.k_{0}=6.79 \mathrm{rad} / \mu \mathrm{m}\right),(\mathrm{b})$ upper $\operatorname{SLR}\left(k_{/ /}=0, k_{0}=7.12 \mathrm{rad} / \mu \mathrm{m}\right),(\mathrm{c})$ lower $\operatorname{SLR}\left(k_{/ /}=1.26 \mathrm{rad} / \mu \mathrm{m}, k_{0}=6.22 \mathrm{rad} / \mu \mathrm{m}\right)$, and (d) upper SLR $\left(k_{/ /}=1.26 \mathrm{rad} / \mu \mathrm{m}, k_{0}=7.65 \mathrm{rad} / \mu \mathrm{m}\right)$.

characteristic simulated near-field profile, with lobes going to the left (toward negative $x$ ) as $z$ increases. In addition, a nonzero (but constant) amplitude is observed in the far-field, corresponding to radiation by order 0 in the $+x$ direction, which we also observe in the simulations (not visible in Figure $7 b)$.

For the upper SLR there is a qualitative change of both the near- and far-field pattern as $k_{/ /}$increases, as seen in the simulations (Figure 7a). In the plane wave picture this can be interpreted via a shift of the three orders with $k_{/ /}>0$. We plot the same amplitudes $\left(a_{0}, a_{+1}, a_{-1}\right)=(0.2,1,1)$, but now for $k_{/ /}=$ $1.26 \mathrm{rad} / \mu \mathrm{m}$ with $k_{0}=7.65 \mathrm{rad} / \mu \mathrm{m}$ in Figure $9 \mathrm{~d}$, to compare with Figure $7 \mathrm{a}$. The near-field shows a bending of the lobes toward $+x$ in the opposite direction as the lower SLR. The farfield is again nonzero, but it has a nonconstant profile, depending on $x$ and $z$, unlike the constant value in the case of $k_{/ /}=0$. This is a consequence of order -1 moving into the light cone, leading to two radiative plane waves (order 0 and -1 ) interfering in the far-field with a nonevanescent lobe toward $-x$. The plane wave model provides a consistent picture with the simulated Bloch modes and can be instrumental in interpreting the near- and far-fields of SLRs. The knowledge of the field amplitude is crucial, for example for the interaction with localized emitters or absorbers.

\section{CONCLUSION}

We have shown how the spectral properties of localized surface plasmon resonances coupled to Rayleigh anomalies influence the properties of the mixed states: surface lattice resonances. The SLR dispersion, line width, and associated stop-gap can be tuned by controlling the energy detuning between the LSPR 
and RAs. In turn, the LSPR spectral characteristics can be tuned via a single geometrical parameter of the nanorods: their width. We have also observed that both the radiative loss and the nearfield confinement (and thus ohmic loss) of the SLRs are enhanced as the LSPR energy approaches the RA energy. The radiative component of the upper SLR displays two regimes depending on in-plane momentum. In the low-momentum regime before crossing with the $(-1,0)$ RA resonance, the upper SLR has only one radiation loss route. In the higher momentum regime, there are two radiation loss channels for the upper SLR due to the $(-1,0)$ diffraction order entering the regime above the light line. This second radiation channel induces a cusp in the radiative loss portion and an optimum point at a nonzero $k_{/ /}$. In contrast, the lower SLR is simpler with always only one radiative channel, as it becomes less confined and more radiative as $k_{/ /}$increases. Additionally, these properties are qualitatively interpreted with a plane wave model, explaining the evolution of the SLRs via the amplitude and radiative character of their Fourier components. With the plane wave model picture, the upper SLR field profile evolution can be qualitatively explained via a change in the radiative character of a Fourier component.

Our results demonstrate the vast possibilities to tailor the properties of hybrid plasmonic/photonic modes by simple geometrical design and provide insight into the interplay between plasmonic resonances and diffraction in arrays of metallic nanoparticles. The results show the interesting balance between diffraction orders, which can be exploited to control both the near- and far-field profile for various applications.

\section{AUTHOR INFORMATION}

\section{Corresponding Authors}

*E-mail: aimi.abass@elis.ugent.be.

*E-mail: s.rodriguez@amolf.nl.

\section{Author Contributions}

${ }^{\#}$ A. Abass and S.R.K Rodriguez contributed equally.

\section{Notes}

The authors declare no competing financial interest.

\section{ACKNOWLEDGMENTS}

We thank Bas Ketelaars for the fabrication of the nanorod arrays and Olaf Janssen for stimulating discussions. This work is supported by the Flemish IWT-SBO project SiLaSol (No. 3E100243), the Interuniversity Attraction Poles program of the Belgian Science Policy Office under Grant No. IAP P7-35 photonics@be, The Netherlands Foundation for Fundamental Research on Matter (FOM), and The Netherlands Organization for Scientific Research (NWO), and is part of an industrial partnership program between Philips and FOM.

\section{REFERENCES}

(1) Bohren, C. F.; Huffman, D. Absorption and Scattering of Light by Small Particles; Wiley Science, 1983.

(2) Lochbihler, H. Surface polaritons on gold-wire gratings. Phys. Rev. B 1994, 50, 4795-4801.

(3) Barnes, W. L.; Preist, T. W.; Kitson, S. C.; Sambles, J. R. Physical origin of photonic energy gaps in the propagation of surface plasmons on gratings. Phys. Rev. B 1996, 54, 6227-6244.

(4) Kitson, S. C.; Barnes, W. L.; Sambles, J. R. Full photonic band gap for surface modes in the visible. Phys. Rev. Lett. 1996, 77, 26702673.
(5) Ghoshal, A.; Divliansky, I.; Kik, P. G. Experimental observation of mode-selective anticrossing in surface-plasmon-coupled metal nanoparticle arrays. Appl. Phys. Lett. 2009, 94, 171108.

(6) Martín-Moreno, L.; García-Vidal, F. J.; Lezec, H. J.; Pellerin, K. M.; Thio, T.; Pendry, J. B.; Ebbesen, T. W. Theory of extraordinary optical transmission through subwavelength hole arrays. Phys. Rev. Lett. 2001, 86, 1114-1117.

(7) Krishnan, A.; Thio, T.; Kim, T.; Lezec, H.; Ebbesen, T.; Wolff, P.; Pendry, J.; Martin-Moreno, L.; Garcia-Vidal, F. Evanescently coupled resonance in surface plasmon enhanced transmission. Opt. Commun. 2001, 200, 1-7.

(8) Sauvan, C.; Billaudeau, C.; Collin, S.; Bardou, N.; Pardo, F.; Pelouard, J.-L.; Lalanne, P. Surface plasmon coupling on metallic film perforated by two-dimensional rectangular hole array. Appl. Phys. Lett. 2008, 92, 011125.

(9) Billaudeau, C.; Collin, S.; Sauvan, C.; Bardou, N.; Pardo, F.; Pelouard, J.-L. Angle-resolved transmission measurements through anisotropic two-dimensional plasmonic crystals. Opt. Lett. 2008, 33, $165-167$.

(10) Ropers, C.; Park, D. J.; Stibenz, G.; Steinmeyer, G.; Kim, J.; Kim, D. S.; Lienau, C. Femtosecond light transmission and subradiant damping in plasmonic crystals. Phys. Rev. Lett. 2005, 94, 113901.

(11) Lee, K. G.; Park, Q.-H. Coupling of surface plasmon polaritons and light in metallic nanoslits. Phys. Rev. Lett. 2005, 95, 103902.

(12) Taubert, R.; Dregely, D.; Stroucken, T.; Christ, A.; Giessen, H. Octave-wide photonic band gap in three-dimensional plasmonic Bragg structures and limitations of radiative coupling. Nat. Commun. 2012, 3.

(13) Christ, A.; Tikhodeev, S. G.; Gippius, N. A.; Kuhl, J.; Giessen, $\mathrm{H}$. Waveguide-plasmon polaritons: strong coupling of photonic and electronic resonances in a metallic photonic crystal slab. Phys. Rev. Lett. 2003, 91, 183901.

(14) Rodriguez, S. R. K.; Murai, S.; Verschuuren, M. A.; Rivas, J. G. Light-emitting waveguide-plasmon polaritons. Phys. Rev. Lett. 2012, 109, 166803.

(15) Zou, S.; Schatz, G. C. Narrow plasmonic/photonic extinction and scattering line shapes for one and two dimensional silver nanoparticle arrays. J. Chem. Phys. 2004, 121, 12606-12612.

(16) Hicks, E. M.; Zou, S.; Schatz, G. C.; Spears, K. G.; Van Duyne, R. P.; Gunnarsson, L.; Rindzevicius, T.; Kasemo, B.; Käll, M. Controlling plasmon line shapes through diffractive coupling in linear arrays of cylindrical nanoparticles fabricated by electron beam lithography. Nano Lett. 2005, 5, 1065-1070.

(17) García de Abajo, F. J.; Sáenz, J. J. Electromagnetic surface modes in structured perfect-conductor surfaces. Phys. Rev. Lett. 2005, 95, 233901.

(18) García de Abajo, F. J. Colloquium: light scattering by particle and hole arrays. Rev. Mod. Phys. 2007, 79, 1267-1290.

(19) Chu, Y.; Schonbrun, E.; Yang, T.; Crozier, K. B. Experimental observation of narrow surface plasmon resonances in gold nanoparticle arrays. Appl. Phys. Lett. 2008, 93, 181108.

(20) Auguié, B.; Barnes, W. L. Collective resonances in gold nanoparticle arrays. Phys. Rev. Lett. 2008, 101, 143902.

(21) Kravets, V. G.; Schedin, F.; Grigorenko, A. N. Extremely narrow plasmon resonances based on diffraction coupling of localized plasmons in arrays of metallic nanoparticles. Phys. Rev. Lett. 2008, $101,087403$.

(22) Vecchi, G.; Giannini, V.; Gómez Rivas, J. Surface modes in plasmonic crystals induced by diffractive coupling of nanoantennas. Phys. Rev. B 2009, 80, 201401.

(23) Rodriguez, S. R. K.; Abass, A.; Maes, B.; Janssen, O. T. A.; Vecchi, G.; Gómez Rivas, J. Coupling bright and dark plasmonic lattice resonances. Phys. Rev. X 2011, 1, 021019.

(24) Zhou, W.; Odom, T. W. Tunable subradiant lattice plasmons by out-of-plane dipolar interactions. Nat. Mater. 2011, 6, 423-427.

(25) Teperik, T. V.; Degiron, A. Design strategies to tailor the narrow plasmon-photonic resonances in arrays of metallic nanoparticles. Phys. Rev. B 2012, 86, 245425. 
(26) Rodriguez, S.; Schaafsma, M.; Berrier, A.; Rivas, J. G. Collective resonances in plasmonic crystals: size matters. Phys. B: Condensed Matter 2012, 407, 4081-4085.

(27) Vecchi, G.; Giannini, V.; Gómez Rivas, J. Shaping the fluorescent emission by lattice resonances in plasmonic crystals of nanoantennas. Phys. Rev. Lett. 2009, 102, 146807.

(28) Rodriguez, S. R. K.; Lozano, G.; Verschuuren, M. A.; Gomes, R.; Lambert, K.; Geyter, B. D.; Hassinen, A.; Thourhout, D. V.; Hens, Z.; Rivas, J. G. Quantum rod emission coupled to plasmonic lattice resonances: A collective directional source of polarized light. Appl. Phys. Lett. 2012, 100, 111103.

(29) Lozano, G.; Louwers, D. J.; Rodriguez, S. R. K.; Murai, S.; Jansen, O. T.; Verschuuren, M. A.; Gomez Rivas, J. Plasmonics for solid-state lighting: enhanced excitation and directional emission of highly efficient light sources. Light Sci. Appl. 2013, 2, e66.

(30) Zhou, W.; Dridi, M.; Suh, J. Y.; Kim, C. H.; Co, D. T.; Wasielewski, M. R.; Schatz, G. C.; Odom, T. W. Lasing action in strongly coupled plasmonic nanocavity arrays. Nat. Nanotechnol. 2013, $8,506-511$.

(31) Offermans, P.; Schaafsma, M. C.; Rodriguez, S. R. K.; Zhang, Y.; Crego-Calama, M.; Brongersma, S. H.; Gómez Rivas, J. Universal scaling of the figure of merit of plasmonic sensors. ACS Nano 2011, 5, $5151-5157$.

(32) Maier, S. A. Plasmonics: Fundamentals and Applications; Springer: New York, USA, 2007; pp 607-636.

(33) Giannini, V.; Vecchi, G.; Gómez Rivas, J. Lighting up multipolar surface plasmon polaritons by collective resonances in arrays of nanoantennas. Phys. Rev. Lett. 2010, 105, 266801.

(34) Francescato, Y.; Giannini, V.; Maier, S. A. Plasmonic systems unveiled by Fano resonances. ACS Nano 2012, 6, 1830-1838.

(35) Luk'yanchuk, B.; Zheludev, N. I.; Maier, S. A.; Halas, N. J.; Nordlander, P.; Giessen, H.; Chong, C. T. The Fano resonance in plasmonic nanostructures and metamaterials. Nat. Mater. 2010, 9, $707-715$.

(36) Lovera, A.; Gallinet, B.; Nordlander, P.; Martin, O. J. Mechanisms of Fano resonances in coupled plasmonic systems. ACS Nano 2013, 7, 4527-4536.

(37) Engelen, R.; Mori, D.; Baba, T.; Kuipers, L. Subwavelength structure of the evanescent field of an optical Bloch wave. Phys. Rev. Lett. 2009, 102. 